\title{
December 2013 Pulmonary Case of the Month: Natural Progression
}

\author{
Robert W. Viggiano, MD \\ Department of Pulmonary Medicine \\ Mayo Clinic Arizona \\ Scottsdale, AZ
}

\section{History of Present IIIness}

A 68 year old woman was seen for increased back pain in April 2012. In 2000 she had a right lower lobe lung resection for low grade adenocarcinoma, bronchoalveolar type, nonmucinous. Her mass was $2.6 \mathrm{~cm}$ in maximal dimension extending to but not invading the pleura. There were clear surgical margins but involvement of one bronchial node. Multiple mediastinal nodes were negative. She had back pain for many years and yearly CTs were negative for metastatic disease.

\section{$\mathrm{PMH}, \mathrm{SH}, \mathrm{FH}$}

Other than the above there was no significant past medical history, social history or family history.

\section{Medications}

- Non-steroidal anti-inflammatory drugs for pain

- Nitrofurantoin for chronic urinary tract infections

\section{Physical Examination}

There was tenderness to palpation over the mid-thoracic spine and evidence of a previous thoracotomy.

\section{Laboratory}

Her complete blood count (CBC), urinanalysis, liver function tests, and calcium were all within normal limits.

\section{Radiology}

An x-ray of the chest is interpreted as unchanged from previous $\mathrm{x}$-rays. 
At this point which of the following radiologic testing is not indicated?

1. Bone scan

2. CT scan of the chest

3. Magnetic resonance imaging

4. Serial chest $\mathrm{x}$-rays

5. Thoracic PET scan 


\section{Correct! \\ 4. Serial chest x-rays}

The patient has a history of lung cancer and new or changing back pain should raise a suspicion of metastatic disease, even 12 years after her thoracotomy. Plain x-rays necessitate a $1 \mathrm{~cm}$ diameter mass and $50 \%$ bone mineral loss at minimum for detection. Up to $40 \%$ of lesions will be unidentified by X-rays (1). The other choices are all reasonable for detecting metastatic disease (2). In this case a thoracic CT/PET scan was performed which showed a lesion in T5 (Figure 1, Panel A) which was biopsied (Figure 1, Panel B).
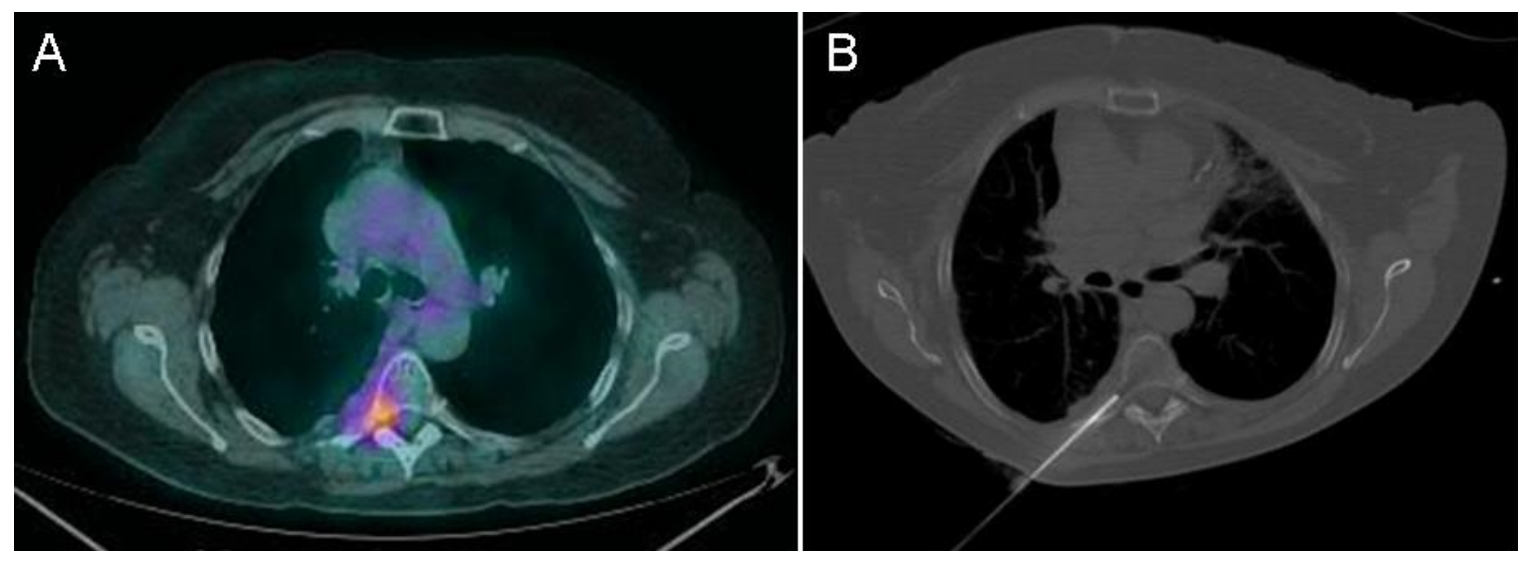

Figure 1. Panel A: CT/PET scan showing increased glucose metabolism in T5. Panel B: biopsy of lesion.

The biopsy showed adenocarcinoma. Immunochemical staining for thyroid transcription factor-1 (TTF-1) and napsin A were positive consistent with metastatic adenocarcinoma of the lung. Epidermal growth factor receptor (EGFR) and anaplastic lymphoma kinase (ALK) were negative. Radiation therapy was recommended.

In addition to the radiation therapy which of the following is the best choice for chemotherapy?

1. Carboplatin + pemetrexed + bevacizumab

2. Carboplatinum alone

3. Cispatin alone

4. Cisplatin + pemetrexed

5. Cisplatin + pemetrexed + crizotinib 


\section{Correct! \\ 4. Cisplatin + premetrexed}

Premetrexed plus a platinum-based antineoplastic agent such as cisplatin or carboplatin is considered to be the first-line therapy for non-small cell lung cancer (3). Addition of EGFR receptor monoclonal antibodies can improve survival in EGFR+ lung cancers. Similarly, crizotinib, an ALK inhibitor, can be added for ALK + lung cancers. The patient was treated with radiation therapy which was completed on 6/7/12. Radiation therapy was followed by 4 cycles of cisplatinum plus pemetrexed concluding in September 2012. She was seen in October 2012 and doing well. However, in late December 2012 she was having nonspecific aches and pains and some shortness of breath. She received amoxicillin for a few days with minimal improvement. The nitrofurantoin was discontinued.

Which of the following are diagnostic considerations as a cause of her shortness of breath?

1. Chemotherapy-induced lung disease

2. Lymphangitic spread of lung cancer

3. Nitrofurantoin lung

4. Pneumonia

5. All of the above 


\section{Correct!}

\section{All of the above}

The differential diagnosis of shortness of breath in this situation is large and includes pneumonia from typical bacteria such as Streptococcus pneumoniae, atypical pneumonia in an immunocompromised host; complications of drugs and/or radiation; progression of underlying lung cancer; or a variety of other causes not related to her underlying disease. A repeat thoracic CT scan was performed and compared to a previous CT scan from October 2012 (Figure 2).

$10 / 5 / 12$ $1 / 3 / 13$

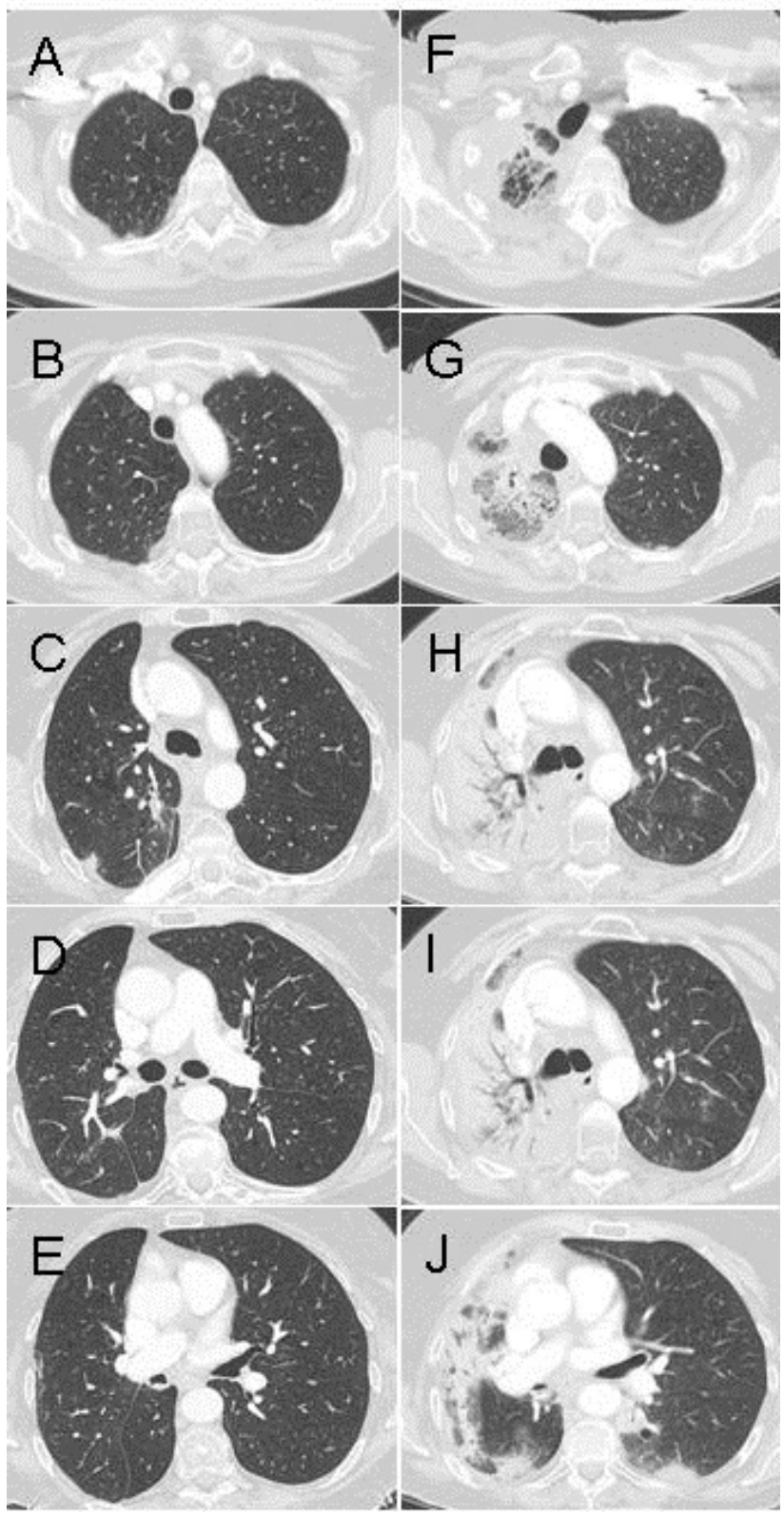

Figure 2. Selected views from thoracic CT scans done 10/5/13 (A-E) and corresponding views from $1 / 3 / 13(\mathrm{~F}-\mathrm{J})$. 
Which of the following should be done next?

1. ${ }^{18}$ FDG-PET scanning

2. Bronchoscopy with transbronchial biopsy

3. Open surgical lung biopsy

4. Percutaneous transthoracic needle biopsy

5. Serial imaging to assess for change 


\section{Correct! \\ 2. Bronchoscopy with transbronchial biopsy}

Bronchoscopy with transbronchial biopsy is the most appropriate next step among the choices listed. A case could be made for choice 3. Open surgical lung biopsy. However, video-assisted thoracoscopic surgical biopsy is generally favored over the open form of the procedure for the diagnosis of interstitial lung abnormalities. Furthermore, a surgical lung biopsy may not be required at all if the less morbid and less expensive bronchcoscopic biopsy establishes a diagnosis. Serial imaging to assess for change is not appropriate given what is apparently an acute and potentially serious abnormality. ${ }^{18}$ FDG-PET scan would likely not be management-altering in this case. Even if tracer accumulation was seen within the pulmonary opacities at ${ }^{18}$ FDG-PET, that finding would be nonspecific and a tissue diagnosis would be pursued regardless. Needle biopsy offers no advantage over bronchoscopy and has a higher complication rate.

The patient did consent to bronchoscopy but refused transbronchoscopic biopsy. Bronchoscopy with bronchoalveolar lavage was performed 1/7/13. Cultures were negative and cytology was negative for malignancy.

\section{What should be done next?}

1. Begin bevacizumab for probable lympangitic spread of her lung cancer

2. Corticosteroids

3. Empiric amphotericin B for Aspergillus pneumonia

4. Empiric piperacillin and tazobactam for community-acquired pneumonia

5. Empiric sulfamethoxazole/trimethoprim for Pneumocystis jiroveci 


\section{Correct! \\ 2. Corticosteroids}

Although the diagnosis is not confirmed, the best working diagnosis is radiation pneumonitis with a possibility of nitrofurantoin lung. The treatment for both is removal of the stimulus with addition of corticosteroids (4). Nitrofurantoin was already stopped on $1 / 3 / 13$. Bronchoalveolar lavage is reasonably good at excluding infection and carcinoma. Radiation pneumonitis would best fit the patient's pneumonitis in the absence of infection and metastatic cancer. However, the patient refused corticosteroids but did consent to follow-up. She returned 18 days later on 1/25/13 complaining of increasing shortness of breath. A repeat chest $x$-ray was performed (Figure 3 ).

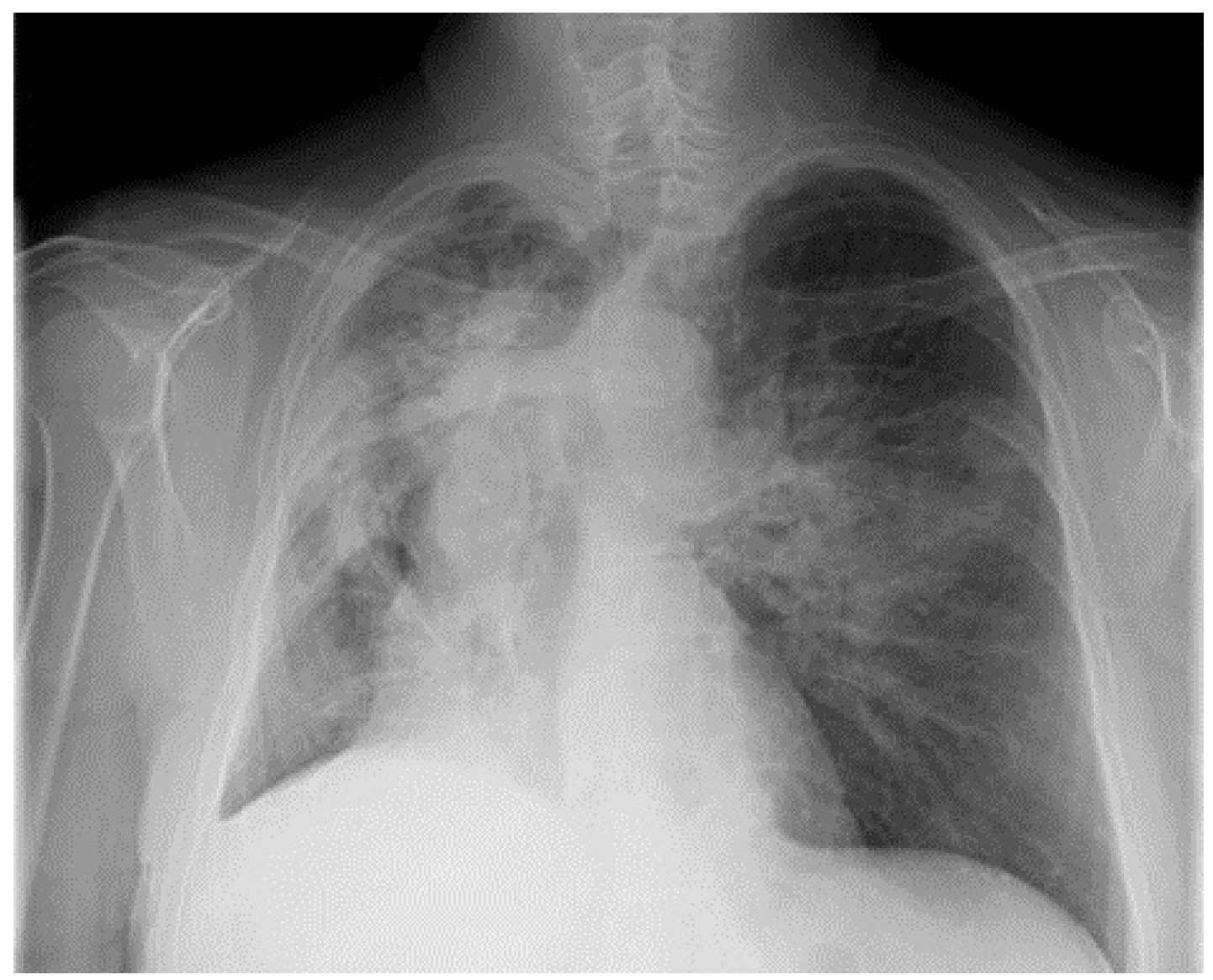

Figure 3. Repeat chest $x$-ray on $1 / 25 / 13$ showing progression of bilateral consolidation.

What should be done next?

1. Azathioprine

2. Cyclosporine

3. Nonsteroidal anti-inflammatory drugs

4. Re-recommend corticosteroids

5. Tacrolimus 


\section{Correct! \\ 4. Re-recommend corticosteroids}

Corticosteroids are commonly used for the treatment of radiation pneumonitis (4). Prednisone is started at $1 \mathrm{mg} / \mathrm{kg} /$ day upon diagnosis and is continued for several weeks and then tapered slowly. Other agents that have been used for treatment of radiation pneumonitis include azathioprine and cyclosporin A but there is limited experience with these medications.

The patient had relief of her dyspnea and serial chest x-rays (Figure 4) and a CT scan (Figure 5) showed improvement in her lung consolidation.
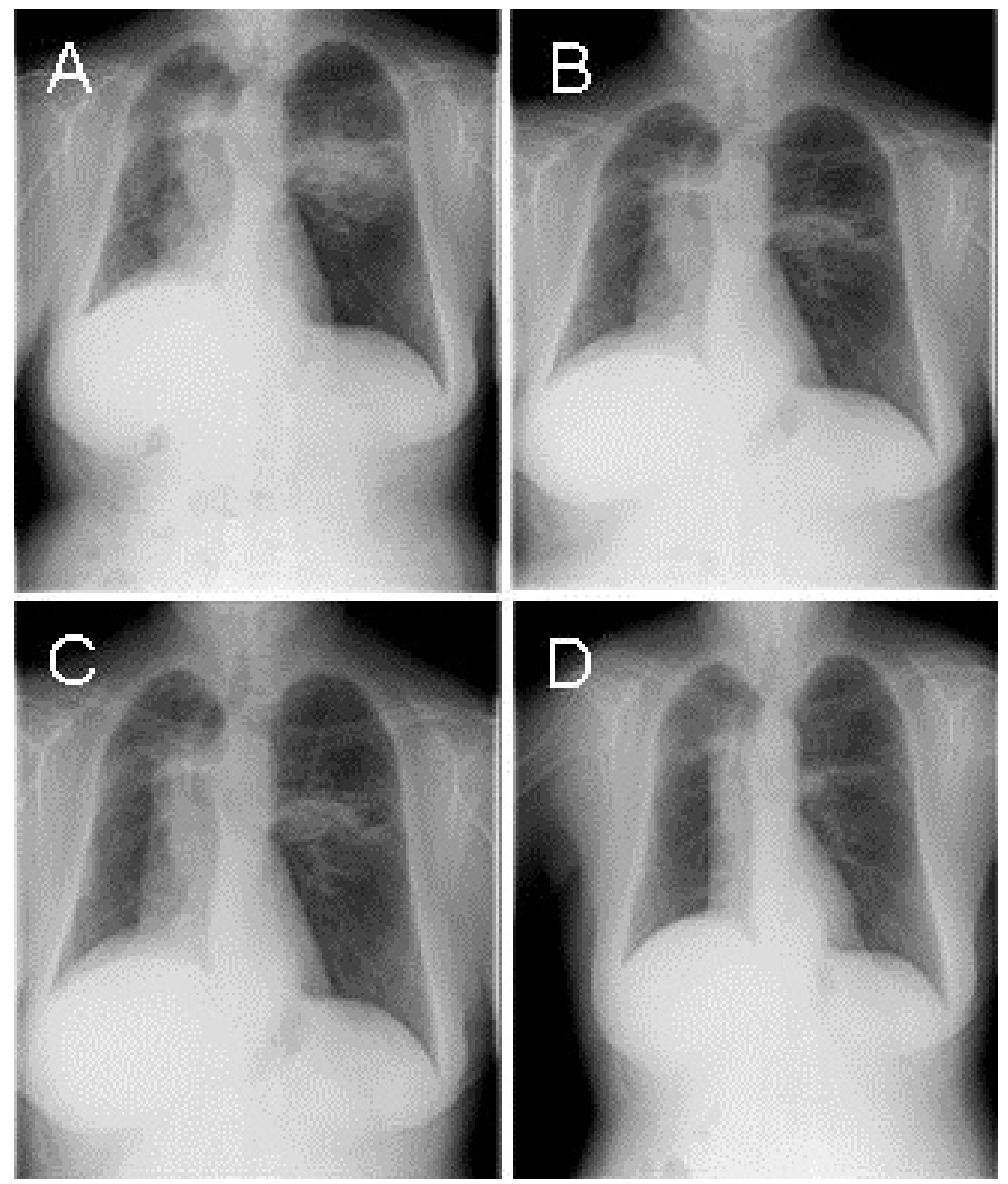

Figure 4. Serial chest $x$-rays from 1/30/13 (A), 2/6/13 (B), 2/20/13 (C) and 5/6/13 (D). 


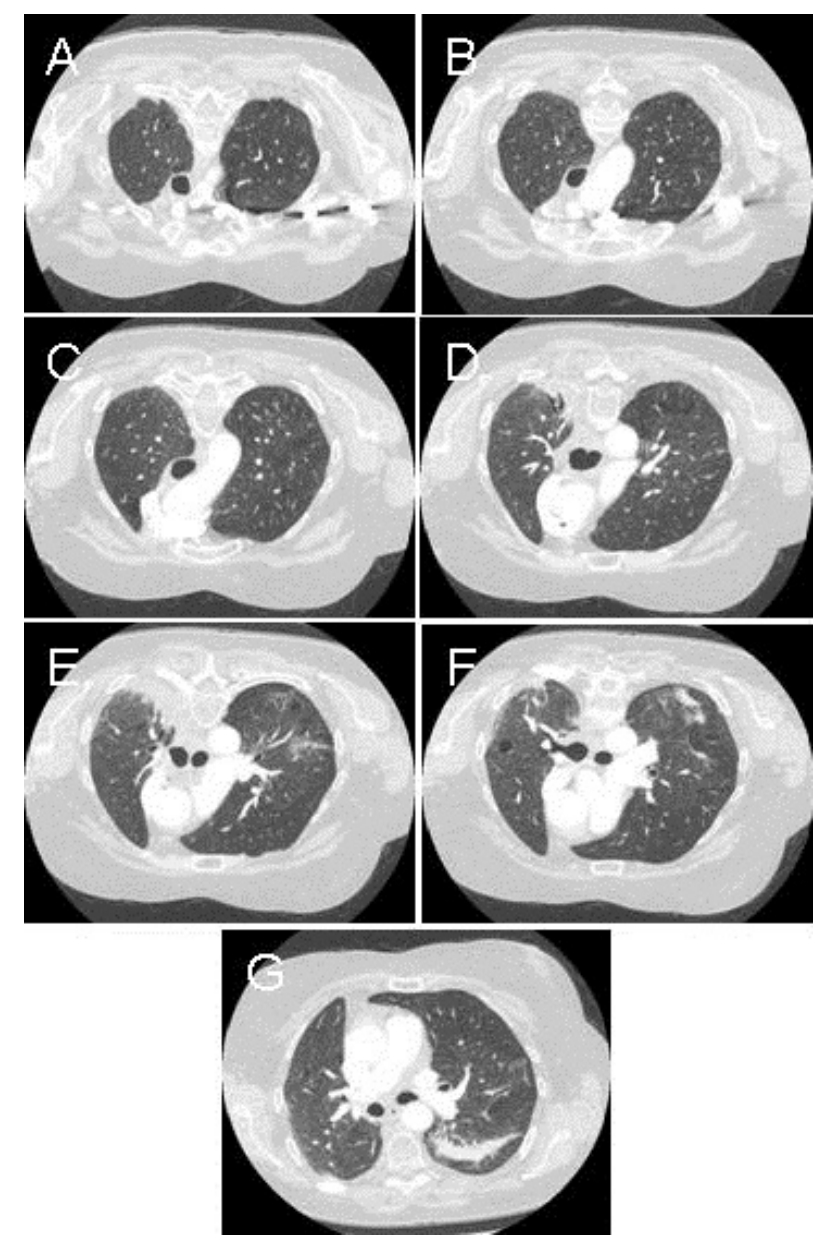

Figure 5. Selected lung windows from a thoracic CT scan performed on 5/6/13.

It is unclear what role nitrofurantoin played in the patient's overall illness. It is possible that the combination of the nitrofurantoin may have exacerbated the radiation pneumonitis although a PubMed search revealed no reports.

\section{References}

1. Salvo N, Christakis M, Rubenstein J, de Sa E, Napolskikh J, Sinclair E, Ford $\mathrm{M}, \mathrm{Goh} \mathrm{P}, \mathrm{Chow} \mathrm{E}$. The role of plain radiographs in management of bone metastases. Journal of Palliative Medicine. 2009;12(2):195-8. [CrossRef] [PubMed]

2. Shah LM, Salzman KL. Imaging of spinal metastatic disease. Int J Surg Oncol. 2011;2011:769753.

3. Ettinger DS et al. NCCN Clinical Practice Guidelines in Oncology: Non-small Cell Lung Cancer V.2.2009. Available at: http://misc.medscape.com/images/586/420/nscl.pdf (accessed 11/8/13).

4. Almuete VI. Treatment of radiation pneumonitis. Medscape. 2003. Available at: http://www.medscape.com/viewarticle/446886 (accessed 11/8/13). 